\title{
Toxicogenomics and its application in cancer pathogenesis
}

\author{
Kapoor R.A. ${ }^{1}$, Ladak S.S. ${ }^{1}$, Gomase V.S. ${ }^{2}$ \\ ${ }^{1}$ Padmashree Dr. D.Y. Patil University, Navi Mumbai, 400614, India \\ ${ }^{2}$ School of Technology, S.R.T.M. University, Sub-Centre, Latur, 413512, India
}

\begin{abstract}
Toxicology, the study of poisons, focuses on substances and treatments that cause adverse effects in living things. A critical part of this study is the characterization of the adverse effects at the level of the organism, the tissue, the cell, and the molecular makeup of the cell. Thus, Toxicology traditionally has focused on phenotypic changes in an organism that result from exposure to chemical, physical, or biologic agents. Such changes range from reversible effects, such as transient skin reactions, to chronic diseases, such as cancer, to the extreme end point of death. Typical whole-animal toxicology studies may range from single-dose acute to chronic lifetime exposures, and they include assessments of end points such as clinical signs of toxicity, body and organ weight changes, clinical chemistry, and histopathologic responses.
\end{abstract}

\section{INTRODUCTION}

Toxicogenomic technologies comprise several different technology platforms for analysis of genomes, transcripts, proteins, and metabolites. It is important to recognize two additional issues associated with the use of toxicogenomic technologies. First, the large quantity of information that a single experiment can generate, and the comprehensive nature of this information, is much greater than what traditional experiments generate. Second, the advancement of computing power and techniques enable these large amounts of information to be synthesized from different sources and experiments and to be analyzed in novel ways[1]. Genomic technologies encompass both genome sequencing technologies, which derive DNA sequences from genes and other regions of DNA, and genotype analysis, which detects sequence variations between individuals in individual genes. The convergence of genome sequencing and genotyping technologies will eventually enable whole-genome sequences of individuals to be analyzed [2]. Transcriptomic technologies (or gene expression profiling) measure mRNA expression in a highly parallel assay system, usually using microarrays. As the first widely available method for global analysis of gene expression, DNA microarrays are the emblematic technology of the post-genomic era. Microarray technology for transcriptomics has enabled the analysis of complex, multigene systems and their responses to environmental perturbations [3]. Proteomics is the study of collections of proteins in living systems. Because the same proteins may exist in multiple modified and variant forms, proteomes are more complex than the genomes and transcriptomes that encode them. Proteomic technologies use mass spectrometry (MS) and microarray technologies to resolve and identify the components of complex protein mixtures, to identify and map protein modifications, to characterize protein functional associations, and to compare proteomic changes quantitatively in different biologic states [4]. Bioinformatics is a branch of computational biology focused on applying advanced computational techniques to the collection, management, and analysis of numerical biologic data. Elements of bioinformatics are essential to the practice of all genomic technologies. Bioinformatics also encompasses the integration of data across genomic technologies, the integration of genomic data with data from other observations and measurements, and the integration of all these data in databases and related information resources. It is helpful to think of bioinformatics not as a separate discipline but as the universal means of analyzing and integrating information in biology [5]. Metabolomics is a systems approach for studying in vivo metabolic profiles, which promises to provide information on drug toxicity, disease processes and gene function at several stages in the discovery and development process. A key strength of metabolomic approaches is that they can be used to noninvasively and repeatedly measure changes in living tissues and living animals and that they measure changes in the actual metabolic flow. As with proteomics, the major limitation of metabolomics is the difficulty of comprehensively measuring diverse metabolites in complex biologic systems [6]. 


\section{PHARMACOLOGY OF TOXICOGENOMICAL GENE THERAPY}

Pharmacology is the study of drug action. More specifically, it is the study of the interactions that occur between a living organism and exogenous chemicals that alter normal biochemical function. If substances have medicinal properties, they are considered pharmaceuticals. The field encompasses drug composition and properties, interactions, toxicology, therapy, and medical applications and antipathogenic capabilities [7].There have been various advances in the field of study of drug action, for example genotype-phenotype correlation in $\beta$-thalassemia patients (both major and intermedia) was observed and it was concluded that treatment with pharmacological agents like hypoxyurea lead to the increased synthesis of globin which indirectly resulted in elevated levels of haemoglobin $\mathrm{F}(\mathrm{HbF})$ that decreased the severity of sickle cell anaemia and $\beta$ thalassemia [8]. Human exposure to this toxic metalloid is long known to result in hazardous health outcomes, including cancers of skin and other internal organs. Although skin lesions are recognized as the most sensitive end points of chronic arsenicism, only $15-20 \%$ of the exposed individuals show arsenic-induced skin lesion. Hence, it is of prime interest to identify the susceptible factors through gene expression, that render one group susceptible to arsenic toxicity, while others remain skin-asymptomatic even after exposure at a similar extent. It was identified a unique repertoire of genes that are overexpressed inarsenic exposed individuals with skin lesions but not in other groups. Hence identification of these genes play an important role in pathogenesis od arsenic induced skin lesion [9].

\section{IMMUNOLOGY OF TOXICOGENOMICS}

Studying gene expression changes associated with immunotoxic processes is a relatively new approach in the field of immunotoxicology. The technique that helps in studying the gene expression is microarray analysis. The basic principle of microarray technology is the hybridization between nucleic acids, one of which is immobilized on a solid matrix. Per gene, a single probe or a few different probes are generated, using either PCR-amplified complementary DNA (cDNA) or synthetic DNA segments (oligonucleotides or oligos) devised on the basis of these cDNA sequences. Labeling of nucleic acid is followed by hybridization and washing and then finally measuring the fluorescence [10]. Microarray technology has been employed in immunology to study molecular functions associated with immunerelated genes in order to better understand immune function and regulation, also referred to as 'immunomics' [11]. Hexachlorobenzene (HCB) is a persistent environmental pollutant with toxic effects in man and rat. Reported adverse effects include hepatic porphyria, toxic effects on the immune and reproductive system, and neurotoxicity. The brown norway (BN) rat strain is very susceptible to HCB-induced immunopathology. To gain more insight into the molecular mechanisms of HCBinduced toxicity, gene expression profiling was performed. Microarray analysis proved to be a suitable tool to reveal changes in gene expression that are consistent with a number of the known (immuno) toxicological effects of HCB in the BN rat and its induction of enzymes involved in metabolism and reproduction [12]. Another example is the mycotoxin deoxynivalenol (DON) which was administered in mice in an experiment. microarray analysis in spleens of mice two hours after exposure to DON or vehicle showed alterations in genes involved in immunity, inflammation, and chemotaxis that are likely to contribute to DON's immunological effects [13].

The effects of a low and a high concentration of the toxic metal cadmium were investigated in an immature T-cell line at an early and late time point using cDNA microarray slides measuring 1,455 genes. Effects on the gene expression proWle were time- and dose-dependent and associated with cell function, cell differentiation, malignant transformation, and cell death [14]. Cholera toxin (CT), is not only responsible for the clinical symptoms of cholera but also a powerful mucosal adjuvant. The latter effect is partly caused by enhancement of the production of various cytokines and suppression of IL-12 expression, stimulating the development of Th2 cells.As expected, expression of Th1 markers was downregulated whereas Th2 markers were 
upregulated. The gene expression profiles were compared with those induced by an activator and an inhibitor of adenylate cyclase, since this enzyme is activated by CT resulting in intracellular cAMP accumulation. Overlap and differences of gene expression alterations induced by these three compounds yielded insight into the involvement of cAMP in CT toxicity [15]. The opportunities that toxicogenomics offers for in vitro (pre-)screening for immunotoxicity are certainly worth further exploitation. When the standardization and validation issues are solved, implementation of transcript, protein, and metabolite profiling (within existing initiatives) in the assessment of immunotoxicity will eventually result in improvement of mechanistic understanding of immunotoxicity and hazard identification of existing and novel compounds [16].

\section{DRUG RESISTANCE MECHANISMS AND CARCINOGENICITY}

The study of biologic mechanisms is a priority for basic and clinical researchers interested in elucidating the cellular, biochemical, and molecular basis of chemical and drug toxicity. Mechanistic insight is required for in-depth understanding of the pathobiology that underlies the adverse response to chemical exposures as well as the development of pharmacologic and nonpharmacologic strategies that can control or contain adverse outcomes in chemical toxicity . From a practical perspective, knowledge and insight gained from mechanistic toxicology investigations have proven useful in risk assessment and drug development [17]. Resistance to treatment with anticancer drugs results from a variety of factors including individual variations in patients and somatic cell genetic differences in tumors, even those from the same tissue of origin. Frequently resistance is intrinsic to the cancer, but as therapy becomes more and more effective, acquired resistance has also become common. Studies on mechanisms of cancer drug resistance have yielded important information about how to circumvent this resistance to improve cancer chemotherapy and have implications for pharmacokinetics of many commonly used drugs [18]. Malignant melanomas, characterized by their high capacity for invasion and metastasis, are one of the most frequent forms of skin cancer. Superficial spreading melanoma is

the most common type of melanoma and grows outwards at first to form an irregular pattern on the skin with an uneven color. It tends to start by spreading out across the surface of the skin, known as the radial growth phase. Nodular melanoma occurs most often on the chest or back. It tends to grow deeper into the skin - quite deeply if it is not removed [19]. Toxicogenomics tools were used to study the genotoxic effect of active compounds on the gene-expression profile of A375 human malignant melanoma cells, through the other molecular functions of target genes, regulatory pathways and mechanisms of malignant melanomas. It also includes the current systems biology approaches, which are very useful for analyzing the biological system and understanding the entire mechanisms of malignant melanomas [20] . Fractal analytical approach in cancer cell investigation provided meaningful insights into the relationships between morphology and phenotype. Recently, some reports have demonstrated that changes in cell shape precede and trigger dramatic modifications in both gene expression and enzymatic function. The study was aimed to investigate if modifications in cancer cell morphology are associated to relevant transition in tumour metabolome, analyzed by means of NMRspectroscopy and PCA flux analysis. MCF-7 and MDA-MB-231 breast cancer cells, exposed to an experimental morphogenetic field. Both cell lines recover a more rounded shape, loosing spindle and invasive protrusions, therefore acquiring a quite "normal" morphology. The cell shape "normalization", obtained through manipulation of environmental cues, is followed by a reversion in tumour metabolic phenotype [21].

\section{THERAPEUTIC AND EXPERIMENTAL STRATEGY}

Human inter-alpha-inhibitor proteins (lalp) are endogenous human plasma proteins that function as serine protease inhibitors. Ialp can block the release of sepsis and block the assembly of protective antigen which leads to the stoppage in the intracellular delivery of the anthrax exotoxins, lethal toxin and edema toxin. Thus lalp could represent a novel adjuvant therapy for the treatment of established anthrax infection [22].Another example states that the management of gastric ulcers can be done by reduction or neutralization of gastric acid secretion. Attempts have been made to reduce the gastric mucosal damage by cytoprotective agents without reducing gastric acidity [23]. The cause of gastric ulcer was examined by studying the various experimental models of gastric mucosal damage. Ethanol induced gastric lesions can be 
produced by intragastric administration of various amount $(0.5-2.0 \mathrm{ml})$ of concentrated $(50-100 \%)$ ethanol. Ulcers were examined few hours after ethanol administration [24]. Gastric mucosal damage as a result of treatment with non-steroidal anti-inflammatory drugs (NSAID) is recognized as the most severe reaction to this class of compounds [25]. The administration of NSAID, including indomethacin and acetylsalicylic acid, represents a very simple and effective animal model for studying the mechanisms underlying NSAID induced gastropathy. To reduce this prodrugs were administered such as sulindac which is initially in the inactive form and first has to be metabolized to the active form in order to exert its anti-inflammatory action [26]. In nonstresed rats, only the active sulfide metabolite was responsible for gastric lesions whereas in stressed rats the inactive sulfone metabolite initiated the gastric ulcerogenesis at higher doses [27]. In another article ,efforts to prevent excessive lymphocyte apoptosis during severe infection have focused either on modification of the signal processing system to create an inherent bias against the triggering of cell death pathways or on inhibition of caspase activity to block their execution. Several studies have shown that transgenic mice overexpressing the antiapoptotic protein Bcl-2 were completely protected against lymphocyte apoptosis in T cells and partially protected in B cells after CLP and showed an increase in survival [28]. The finding suggested that protection resulted from the release of cytoprotective or antiinflammatory molecules from Bcl-2overexpressing cells, from an increase in neutrophils at sites of infection, or both. Despite these findings, recent studies that showed a lower death rate after CLP in transgenic mice expressing the antiapoptotic protein Akt in T cells have added further support to the concept that prevention of lymphocyte apoptosis is an independent survival factor in sepsis [29].

BRCA1 and BRCA2 are important for DNA double-strand break repair by homologous recombination and mutations in these genes predispose to breast and other cancers . Poly(ADPribose) polymerase (PARP) is an enzyme involved in base excision repair, a key pathway in the repair of DNA single-strand breaks. It was seen that BRCA1 or BRCA2 dysfunction unexpectedly and profoundly sensitizes cells to the inhibition of PARP enzymatic activity, resulting in chromosomal instability, cell cycle arrest and subsequent apoptosis. This seems to be because the inhibition of PARP leads to the persistence of DNA lesions normally repaired by homologous recombination. These results illustrate how different pathways cooperate to repair damage, and suggest that the targeted inhibition of particular DNA repair pathways may allow the design of specific and less toxic therapies for cancer [30].

\section{OTHER APPLICATIONS}

Toxblot approach allows the selection of the genes to be represented on the array, permitting arrays to be focused on areas of particular interest to mechanistic or investigative toxicology research programs [31]. It has various applications:

\section{Endocrine Disruption}

Ligand-mediated estrogen receptor (ER) activation, followed by activation (or repression) of estrogen receptor - responsive genes has a complex mode of action which is not accurately studied using in vitro screening. Recent research has shown that the DNA sequence in the estrogen response elements within the estrogen responsive genes have a great influence on the estrogen receptor activation [32]. Hence assays that will measure the activation of a single gene product as a marker for estrogenecity will give misleading results. This lead to development of more sophisticated assays such as the combination of suppression subtractive hybridization [33] and cDNA construction has been employed to characterize the differences between ER-positive and -negative cell lines [34].ToxBlot microarrays were used to characterize gene expression changes that took place in cultured cells exposed to natural estrogens (e.g., estradiol), synthetic (e.g., diethyl stilbestrol), and phytoestrogens and pattern of consistent gene expression emerged which then allowed the evaluation of the endocrine disruption at molecular level [35].

Bone Marrow Toxicity of Insulin- Sensitizing Compounds. 
Microarrays have been used to develop novel insulin-sensitizing agents as therapies for noninsulin-dependent diabetes mellitus (NIDDM). First, the existing therapeutic agents for NIDDM, the thiazolidinediones (TZDs), have undesirabletoxic properties that limit their clinical application [36]. Thus, there is an incentive to develop alternative therapeutic agents with a more favorable toxic profile. Second, the TZDs are known to activate a nuclear receptor, the peroxisome proliferator-activated receptor gamma [37] which represents a potential mechanism for both the toxic and therapeutic effects of these compounds, and assists the design and interpretation of array experiments. Third, a reliable in vitro system is used for evaluating adipogenic potential of compounds, using human bone-marrow stromal-cell culture [38].

\section{CONCLUSION}

Toxicogenomics combines transcript, protein and metabolite profiling with conventional toxicology to investigate the interaction between genes and environmental stress in disease causation [45] . The patterns of altered molecular expression that are caused by specific exposures or disease outcomes have revealed how several toxicants act and cause disease. It is seen that oxicology is gradually evolving into a systems toxicology that will eventually allow us to describe all the toxicological interactions that occur within a living system under stress and use our knowledge of toxicogenomic responses in one species to predict the modes-of-action of similar agents in other species [46].

\section{References}

[1] James K. Selkirk and Tennant R.W. (2003) Pure Appl.Chem ;12:2413-2414.

[2] Tomoyuki Shirai and Makoto Asamoto (2003) Pure Appl.Chem; 12:2419 2422.

[3] Kristin Schirmer, Beat B. Fischer, Danielle J. Madureira and Smitha Pillai (2010) Springer ; ISSN: 1618-2650.

[4] Jon B. Klein, Visith Thongboonkerd (2004) Proteomics in Nephrology ;141:1-10.

[5] John L.Markley, Mark E.Anderson (2007) Pacific Symposium on Biocomputing; 12:157168.

[6] Peter Junghans, Thilo Kaehne (2004) The journal of nutrition; 134: 43-47.

[7] Vallance P., Smart T.G. (2006) British journal of pharmacology ;147(S1): S304-S307.

[8] Sudipa Chakravarty, Harpreet Kaur , Ritushree Kukreti, Amit Chakravarty (2008) Springer ;2:341-350.

[9] Pritha Ghosh C., Harrys Kishore J., Akhilesh Pandey, Ashok K. Giri (2008) Springer; 2:341-350.

[10] Duggan D.J., Bittner M., Chen Y., Meltzer P., Trent J.M. (1999), Nat. Genet;. 21 :10-14.

[11] Glynne R.J., Watson S.R. (2001) John Wiley Sep;. 195(1):20-30.

[12] Ezendam J., Staedtler F., Pennings J., Vandebriel R.J., Pieters R., Harleman J.H., Vos J.G. (2004) Environ. Health Perspect.; 782-791.

[13] Kinser S., Jia Q., Li M., Laughter A., Cornwell P., Corton J.C., Pestka J. (2004) J. Toxicol., Environ. Health A; 67: 1423-1441.

[14] Tsangaris G.Th., Botsonis A., Politis I., Tzortzatou-Stathopoulou F. (2002) Toxicology; 178: $135-160$.

[15] Royaee A.R., Mendis C., Das R., Jett M., Yang D.C. (2006) Mol. Immunol.,43: 702-709.

[16] Kirsten A. Baken, Rob J. Vandebriel (2007) ScienceDirect Methods; 41: 132-141.

[17] Emanuel F., Petricoin III., David K. Ornstein (2002) Journal of the National Cancer Institute; 94: 1576-1578.

[18] Michael M. Gottesman (2002) Annual Review of Medicine ; 53: 615-627.

[19] de Braud F., Khayat D., Kroon B.B., Valdagni R., Bruzzi P., Cascinelli N. (2002). Crit. Rev. Oncol. Hematol. ;47:35-63.

[20] Sun-Long Cheng,Rosa Huang-Liu (2007) Pharmacogenomics; ISSN 1462-2416.

[21]D'Anselmi F., Valerio M., Cucina A., Galli L., Proietti S., Dinicola S., Pasqualato A., Manetti C., Ricci G., Giuliani A., Bizzarri M. (2010) Int J Biochem Cell Biol. PMID: 20460170, doi:10.1016/j.biocel.2010.05.002.

[22] Opal S.M., Lim Y.P. (2010) NCBI PMID: 20523269.

[23] Silen W. (1988) Am. J. Physiol.; 255:G395-G402. 
[24] Sandor Szabo (1987) Scandinavian Journal of Gastroenterology; 22: 21-28.

[25] Robert T. Schoen (1988) The American Journal of Medicine; 86: 449-458.

[26] Glavin G.B., Sitar D.S. (1989) Science Direct, 83: 386-389.

[27] Wallace J. L., Keenan C. M. and Granger D. N. (1990) Am J Physiol Gastrointest Liver Physiol 259: G462-G467.

[28] Hotchkiss R.S., Swanson P.E. (1999) J Immunol ; 162:4148-56.

[29] Bommhardt U., Chang K.C. (2004) J Immunol ; 172:7583-91.

[30] Hannah Farmer, Nuala McCabe (2005) Nature ; 434,:917-921.

[31] William D. Pennie, Jonathan D. Tugwood (2000) Toxicological Sciences ;54:277-283.

[32] William D. Penny and Stephen J. Roberts (1999) Science Direct ; 32(6):483-502.

[33] Luda Diatchenko, Andrea G. Nackley (2007) Journal of the International Association for the Study of Pain; 125:216-24.

[34] Yang V.W., LaBrenz S.R. (1999) J Biol Chem; 274(18):12454-60.

[35] Christoph M. Bamberger, Tobias Else (1999) The Journal of Clinical Endocrinology and Metabolism ; 84(11): 4055-4061.

[36] Deldar A., Stevens C.E. (1993) Toxicologic Pathology ; 21(2):231-40.

[37] Michel Rivier, Irina Safonova (1998) Journal of Investigative Dermatology ; 111, 11161121.

[38] Ohguro N., Fukuda M. (1999) Br J Ophthalmol ; 83(9): 1064-1068.

[39] Michael D. Waters, Jennifer M. Fostel (2004) Nature Reviews Genetics 5, 936-948.

[40] Moein Moghimi S., Christy Hunter A. (2005) The FASEB Journal, 19:311-330.

[41] Mattes W.B., Pettit S.D., Sansone S.A., Bushel P.R., Waters M.D. (2004) Environ Health Perspect 112:495-505. doi:10.1289/ehp.6697.

[42] http://www.ncbi.nlm.nih.gov/books/NBK10209/. (2007) Applications of Toxicogenomic Technologies to Predictive Toxicology and Risk Assessment, ISBN-13: 978-0-309-112987, National Academies Press (US).

[43] Sun-Long Cheng, Rosa Huang-Liu, Jin-Nan Sheu, Shui-Tein Chen, Supachok Sinchaikul and Gregory J Tsay (2007) Pharmacogenomics, 8 (8), 1017-1036, doi: 10.2217/14622416.8.8.1017.

[44] Ethan G. Verrite (2009) Drug Resistant Neoplasms, ISBN: 978-1-61324-474-6, Nova Science Publishers.

[45] Sara Shostak (2005) Social Studies of Science, 35: 367-403.

[46] George Orphanides and lan Kimber (2003) Toxicol. Sci., 75 (1): 1-6. doi: 10.1093/toxsci/kfg101.

[47] Boorman G.A., Anderson S.P., Casey W.M., Brown R.H., Crosby L.M., Gottschalk K., Easton M., Ni H., Morgan K.T. (2002) Toxicol Pathol., 30(1):15-27. 